\title{
Outbursts from the secondary component in OJ 287 and the secondary spin-up
}

\author{
P. Pihajoki ${ }^{\mathrm{a}}$
}

Tuorla Observatory, Department of Physics and Astronomy, University of Turku, 21500 Piikkiö, Finland

\begin{abstract}
At the end of March 2012 a prominent optical outburst was observed in the binary black hole system OJ 287. It does not fit the expected sequence of outbursts from the primary component and the bremsstrahlung outbursts from the accretion disk impacts of the secondary component. These occur in a well established pattern repeated with an approximately 12 year interval. In this work we discuss the possibility that the outburst originates from the secondary black hole. The timing of the 2012 outburst relative to the expected sequence would make it a counterpart of the precursor outbursts in 1993 and 2004, which occured before the main pattern of outbursts. If so, it appears that a precursor occurs when the secondary reaches a constant level above the mean accretion disk level of the primary component. It may be that this encounter induces an outburst in the secondary which is nearly as prominent as the outbursts in the expected sequence. The strength of these outbursts depends strongly on the spin of the secondary. In this work we investigate the limits on the secondary spin-up and spin alignment from interaction with the accretion disk of the primary component and its magnetic field.
\end{abstract}

\section{INTRODUCTION}

The blazar OJ 287 at redshift $z=0.306$ has been shown to exhibit optical flares in a double peaked pattern that repeats in intervals of approximately 12 years [1-3]. This led to a development of a series of binary black hole models starting with [1]. The outbursts are not exactly periodic, however, and to satisfactorily explain the outbursts in 2005 and 2007, a strongly relativistic precessing binary black hole model is required [4-7]. In the precessing binary model, the twin optical outbursts are caused by the secondary component impacting the accretion disk of the primary [4]. The impact tears off a shocked cloud of gas that radiates strongly with a bremsstrahlung spectrum [4]. Further outbursts in the repeating sequence originate from the primary due to enhaced accretion rate caused by the tidal effects of the secondary [5-7].

The magnitude of precession in the OJ 287 system has been found to be approximately $39.1^{\circ}$ per cycle [8], and the maximum relative velocity of binary is close to $10 \%$ of the speed of light. This very relativistic nature of the OJ 287 makes it an ideal candidate for testing general relativity and black hole properties [9]. Recently, the black hole nohair theorem [10, see, e.g.] has been validated for the case of the OJ 287 system at an accuracy level of $30 \%[8,11]$.

In addition to the repeating pattern of bremsstrahlung and tidal outbursts, a class of precursor outbursts have also been identified [12-15]. These outbursts, seen in 1993 and 2004, have occured just prior to the outburst pattern starting with the first bremsstrahlung outburst $[12,15]$. Recently, in March 2012, a new outburst was seen. The timing would make it a counterpart of the 1993 and 2004 precursors, as a new outburst pattern is expected to start with the secondary accretion disk impact in mid 2013 leading to the first outburst in late $2015[16,17]$. The precursor outbursts are shown in Figure 1 together with the optical light curve.

a e-mail: popiha@utu.fi
We discuss in this paper a possible model of the precursor outbursts where the outbursts originate from the secondary component of OJ 287. The impacts of the secondary black hole on the accretion disk of the primary tear off gas clouds that form a corona above the geometrically thin and optically thick disk. As the secondary component subsequently passes through this corona it may accrete some of these clouds. These accretion events could lead to jet brightening in the secondary, and thus to brightening of the OJ 287 system as a whole.

We briefly discuss how the precessing binary model allows a maximum secondary brightness that is high enough, assuming the secondary has a high spin. We then demonstrate that the secondary can attain a high spin that is aligned with the spin axis of the primary by interaction with the accretion disk of the primary. We also discuss ways to confirm whether the precursor emission originates from the secondary component. This would allow for a separate detection of the secondary black hole in addition to the primary black hole that is otherwise responsible for the emission of the OJ 287 system.

\section{PRECURSOR BURSTS}

We have calculated the orbit of the black hole binary using the geometric integration scheme in [18] with Post-Newtonian corrections up to third order. Detailed description of the Post-Newtonian correction terms with references, and the orbital parameters of the system can be found in [8]. In [19] it was noted that precursor bursts seem to occur when the secondary is at a height of $z_{c} \sim 4000 \mathrm{AU}$ above the $z=0$ plane of the accretion disk of the primary. To visualize this relation, we plotted the height of the secondary above the accretion disk as a function of time in Figure 2 in units of $z_{c}$ and multiplied by 10. It is evident that the constant height is a good predictor for the precursor outburst events. 


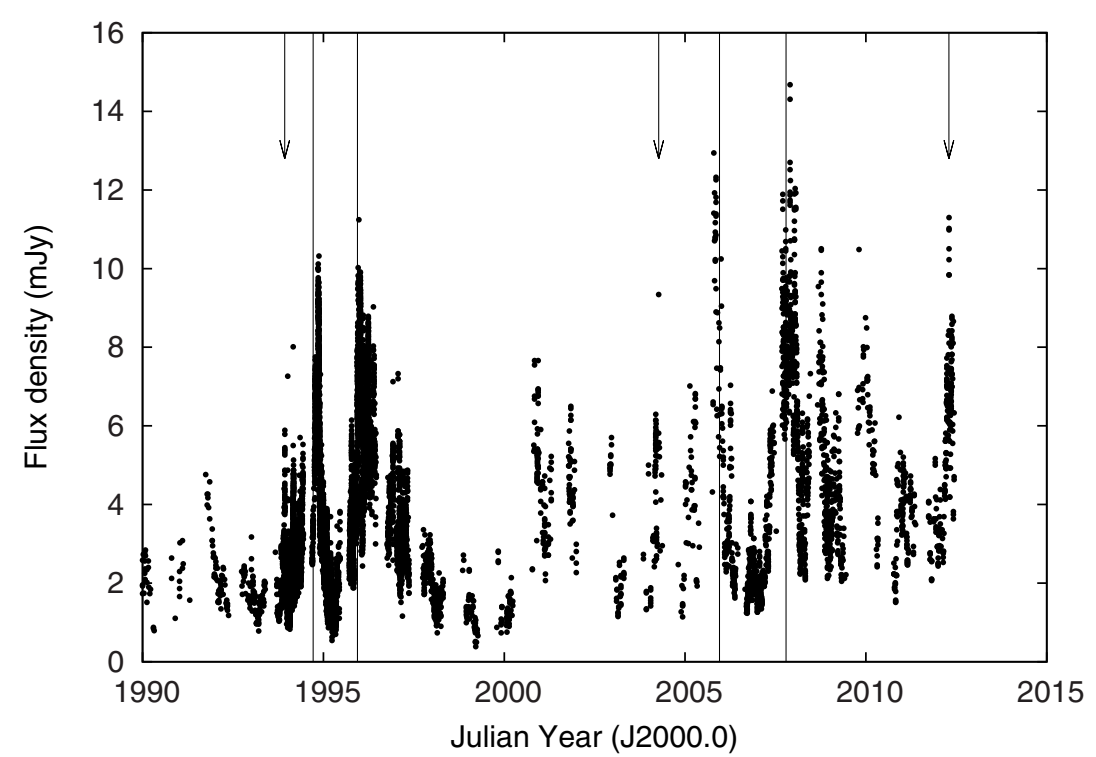

Figure 1. Light curve of OJ 287 in V-band equivalent flux with precursor timings marked with arrows. Outburst timings of the model in [17] are marked with vertical lines.
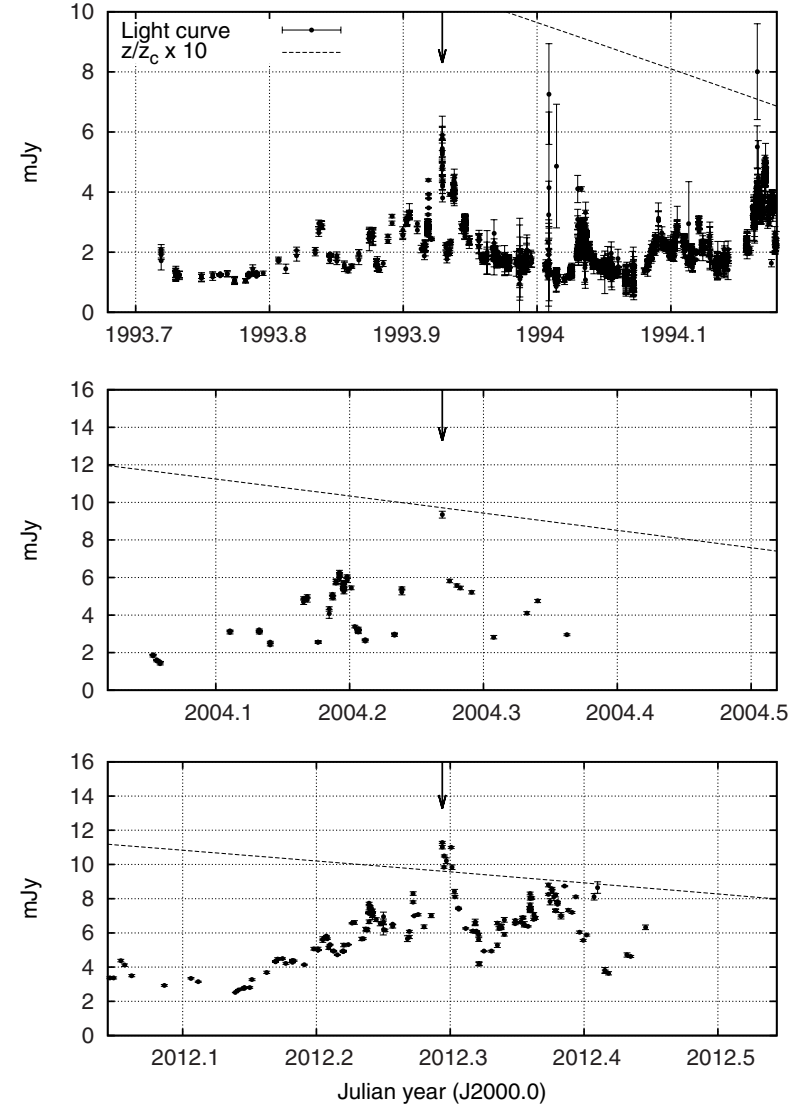

Figure 2. Light curve with error bars near the precursor flares. Flare timings are marked with arrows. The dashed line shows $10 \times$ the height of the secondary above the accretion disk in units of 4084 AU.

These results lead us to a model where the precursor flares arise from the jet brightening of the secondary black hole when it accretes a gas cloud from the corona of the primary accretion disk. These clouds naturally originate as the hot gas bubbles torn off by the secondary from the accretion disk of the primary.

In [19] the coronal clouds and accretion events by the secondary were studied in detail. Assuming the secondary to be maximally spinning, with dimensionless angular momentum $a=J / M^{2} \sim 1$, it was found that the secondary could possibly reach luminosities of $0.25(k / 20)^{-3} L_{\text {Edd }}$, with $k$ related to the maximal expansion of the gas bubbles from the accretion disk impact. The value of $k$ is unknown but it was estimated in [19] to be $\sim 20$. For the secondary $L_{\text {Edd }} \sim 2 \times 10^{46} \mathrm{erg}$, so that the maximum luminosity of the secondary is comparable to the maximum bremsstrahlung emission from the accretion disk impact bubbles, and the maximum jet emission of the primary, accreting at $\sim 10^{-3} \dot{M}_{\text {Edd }}[19]$. Assuming the secondary jet is directed in the same direction as the primary jet, the case for the precursor outbursts originating from the secondary is plausible.

\section{SECONDARY SPIN}

In the previous section it was assumed that the secondary has a high spin, and further that the spins of the secondary and primary are sufficiently aligned. These assumptions are based on the secondary repeatedly interacting with the magnetic fields and gas of the accretion disk of the primary. The angular momentum of the gas in the accretion disk has angular momentum about the impact direction parallel to its angular momentum about the disk axis, albeit in the opposite direction. Thus the gas falling into the secondary can be expected to form a temporary accretion disk [4], the accretion from which in turn would cause the spin of the secondary to increase [20,21]. The direction of the secondary spin axis is affected by the magnetic field of the primary and its accretion disk, in which the orbit of the secondary is embedded [22]. This magnetic field is probably poloidal in nature [23,24], which would also work to bring the spins of the black holes into alignment. 


\subsection{Spin-up}

The rate of the spin-up can be estimated by considering the mass accreted by the secondary during an impact with the accretion disk of the primary. This was estimated in [4] to be some (unknown) fraction of

$$
\Delta m=2 n h \sigma^{2} \sim \Sigma \sigma^{2},
$$

where $n$ is the accretion disk density, $h$ is the accretion disk semiheight, $\Sigma$ is the disk surface density and $\sigma^{2}$ is the interaction cross section. The interaction cross section is $\sigma^{2}=\eta^{2} r_{\text {sch }}^{2}$, where $r_{\text {sch }}$ is the secondary Schwarzschild radius, $\eta=\left(c / v_{\text {rel }}\right)^{2}$ and $v_{\text {rel }}$ is the velocity of secondary relative to the accretion disk. The portion of the mass $\Delta m$ that is not accreted directly ends up either in an expanding coronal cloud [19], in the jet of the primary, back in the accretion disk, or in a temporary accretion disk around the secondary, as proposed in [4]. The timescale for accretion from a temporary accretion disk at Eddington rate from [25] is much longer than the orbital period of the secondary, during which the temporary disk is lost. Thus the temporary accretion disk is unlikely to have much influence in the secondary spin-up. As for the impacts with the accretion disk of the primary, in [19] it was estimated that roughly $\delta m=\Delta m / \eta$ would be accreted during an impact with a coronal cloud. We use the same estimate here for the impact with the accretion disk.

In first approximation, we can look at the matter accreted from the innermost stable circular orbit of the

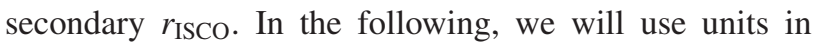
which $G=c=1$. The evolution equations of the black hole mass and spin were first derived in [20], and further elaborated in [21], in which the canonical upper limit $a=0.998$ for a black hole spin was also derived. The equations valid in the region $0 \leq a \leq 1$ are

$$
\begin{aligned}
a & =\sqrt{\frac{2}{3}} \frac{M_{i}}{M}\left(4-\sqrt{18\left(\frac{M_{i}}{M}\right)^{2}-2}\right) \\
\Delta M_{0} & =3 M_{i}\left[\sin ^{-1}\left(\frac{M}{3 M_{i}}\right)-\sin ^{-1}\left(\frac{1}{3}\right)\right],
\end{aligned}
$$

where $M_{i}$ and $M$ are the initial and current mass of the black hole, $a=J / M^{2}$ is the dimensionless black hole spin angular momentum and $\Delta M_{0}$ is the rest mass that has gone down the hole, starting from $a=0$. These equations rest on the assumption that all the matter falling into the black hole has a positive angular momentum (i.e. in the same sense as the black hole spin). This is required due to the fact that the interaction cross section for trapping matter into the hole is greater for matter with an angular momentum in the opposite sense [20]. This requirement is not exactly satisfied in the case of impact with a thin accretion disk. Although the nearly Keplerian shear flow guarantees a net positive angular momentum, always in the same sense regardless of impact distance, the matter in the disk surrounding the impact site doesn't have an uniformly positive angular momentum distribution. Thus, for a more accurate solution, the details of the accretion during the impact would need to taken into account.
An estimate for the time required for a complete spinup of the secondary component in OJ 287 from $a=0$ to $a=1$ now be obtained from the equations (2). When $a=1$ after a time $t$, we have $\Delta M \sim 1.4495 M_{i}=C_{1} M_{i}$ for the increase in the black hole mass and $\Delta M_{0}=1.8464 M_{i}=$ $C_{2} M_{i}$ for the amount of accreted rest mass [21]. Since there are two impacts during each orbital period $P \sim 12 \mathrm{yr}$, we have

$$
\Delta M_{0}=2 \frac{t}{P} \delta m=C_{2} M_{i},
$$

and $M_{i}=M /\left(1+C_{1}\right)$ from which we obtain

$$
t=\frac{C_{2}}{1+C_{1}} \frac{P M}{2 \delta m} \sim 0.38 \frac{P M}{r_{\mathrm{sch}}^{2} \eta \Sigma} .
$$

To estimate $\Sigma$ we use the $\alpha$ disk realization calculated in [4], which was based on the model in [26]. We scale the model according to the equations in [25], since the values $\alpha=1$ for the viscosity and $\dot{M}^{\text {pri }}=0.1 \dot{M}_{\text {Edd }}$ for the accretion rate of the primary used in [4] need to be updated to reflect current knowledge. To estimate the scale factors we first note that a recent study [27] suggests the value of $\alpha$ is approximately in the range $0.1-0.4$, with the high end favored by observations. In line with [19], we take $\alpha=0.5$ from now on. The accretion rate of the primary is also probably not as high as in [4], where the value was based on a luminosity of $3 \times 10^{47} \mathrm{erg}$, as in [28]. However, the emission of OJ 287 is strongly beamed and jet-dominated, and the luminosity of the accretion disk is therefore only a fraction of the total luminosity. In [29] it was suggested that a value of $1.3 \times 10^{46} \mathrm{erg}$ obtained in [30] is more approriate, but still probably an upper limit. This estimated disk luminosity corresponds to an accretion rate of the primary of $\dot{M}^{\text {pri }} \sim 10^{-3}-10^{-2} \dot{M}_{\text {Edd }}$. Using $\dot{M}^{\text {pri }}=$ $10^{-3} \dot{M}_{\text {Edd }}$ we finally get $\Sigma=10^{5} \mathrm{~g} / \mathrm{cm}$, in accordance with [19]. Now, using the value $M^{\text {sec }}=1.4 \times 10^{8}$ for the mass of the secondary [8], and a representative value of $\eta=36$ [19], we find $\delta m \sim 3 \mathrm{M}_{\odot}$ and the estimate for the spin-up time

$$
t \sim 2.0 \times 10^{8} \mathrm{yr} .
$$

Here the effective accretion rate for the secondary $2 \delta m / P \sim 0.5 \mathrm{M}_{\odot} / \mathrm{yr} \sim 0.5 \dot{M}_{\mathrm{Edd}}$. This is possibly excessive, as the secondary accretion during the disk impact is not as significant as in the coronal cloud collisions $[4,19]$, and thus the estimated spin-up timescale is a lower limit.

The estimate is however of same order than the coalescence time of a supermassive black hole binary with parameters like the OJ 287 system, roughly $2 \times 10^{8} \mathrm{yr}$ as obtained from the estimate in [31]. Thus, it is plausible that the secondary in OJ 287 might attain a significant spin via disk interactions by the time it has reached the current inspiral stage.

\subsection{Spin alignment}

Near a spinning black hole the Lense-Thirring precession will tend to twist the inner accretion disk in a plane perpendicular to the black hole spin vector [32]. Correspondingly, through viscous processes, the accretion 
disk effects a torque working to align the black hole spin with the angular momentum vector of the outer accretion disk [33]. Various estimates have been given for the alignment timescale, see e.g. [33-36]. From [34] we have an estimate for the alignment timescale $t_{a}$ as

$$
\begin{aligned}
t_{a}= & 5.6 \times 10^{5} a^{11 / 16}\left(\frac{\alpha}{0.03}\right)^{13 / 8}\left(\frac{L}{0.1 L_{\text {Edd }}}\right)^{-7 / 8} \\
& \times M_{8}^{-1 / 16}\left(\frac{\epsilon}{0.3}\right)^{7 / 8} \mathrm{yr},
\end{aligned}
$$

where $a=J / M^{2}, \alpha$ is the thin disk viscosity parameter $L$ is the luminosity and $\epsilon$ is the efficiency of the accretion process defined as $\epsilon=L /\left(\dot{M} c^{2}\right)$. As was noted in the previous section, if we take $\epsilon=0.3$ and estimate the accretion with $\dot{M}=2 \delta m / P$, we obtain $\dot{M}^{\mathrm{sec}} \sim 0.5 \mathrm{M}_{\odot} / \mathrm{yr}$ $\sim 0.5 \dot{M}_{\text {Edd }}$, so that $L=0.5 L_{\text {Edd }}$. Using the value $\alpha=0.5$ set in the previous section we obtain

$$
t_{a} \sim 1.4 \times 10^{7} a^{11 / 16} \mathrm{yr} .
$$

Thus even for a maximal spin $a \sim 1$, alignment could happen in a rather short time scale compared to the spin-up time or the coalescence time. However, due to the fact that most of the secondary accretion during the disk impact is likely from gas falling directly into the hole as opposed to accretion from a temporary accretion disk, this alignment timescale is a lower limit only.

In addition to accretion of matter with angular momentum, an ambient magnetic field can also effect a torque on the spin of a black hole. An active galactic nucleus like OJ 287 with a jet is very likely to have a significant magnetic field present, rising from the magnetized accretion disk of the primary [37-39]. An equation for the magnetic torque and an estimation for the alignment timescale were first conjectured by Press in [22] and later confirmed in [40]. The magnetic alignment timescale $t_{m}$ for a magnetic field of constant strength $B$ that is assumed uniform far from the black hole was found in [40] to be

$$
t_{m}=10^{36}\left(\frac{M}{\mathrm{M}_{\odot}}\right)^{-1}\left(\frac{B}{10^{-5} \mathrm{G}}\right)^{-2} \mathrm{yr},
$$

where $M$ is the black hole mass. For an estimate of the magnetic field strength in the OJ 287 system that is consistent with the used accretion disk model of [26], we use the approximation from [25]

$$
B \leq 1.9 \times 10^{1} 0 \alpha^{1 / 10} m^{-2 / 5} \dot{m}^{-4 / 5} \tilde{r}^{-9 / 5}\left(1-\tilde{r}^{-1 / 2}\right)^{4 / 5} \mathrm{G},
$$

where $m$ is the black hole mass in solar masses, $\dot{m}$ is the accretion rate in units of Eddington accretion rate and $\tilde{r}$ is the radial distance in units of $3 r_{\text {sch. }}$. In the case of OJ 287 we have $\tilde{r} \sim 10$, and we find $B \leq 4.1 \times 10^{6} \mathrm{G}$. This gives an alignment timescale $t_{m} \sim 4 \times 10^{4} \mathrm{yr}$ for the secondary black hole. So in a first approximation $t_{m} \ll t_{a}$, and the magnetic field is likely to be a major influence in both the secondary spin direction as well as the directing its jet as shown in $[41,42]$.

\section{DISCUSSION}

One way to verify the presence of the secondary jet in the radiation of OJ 287 would be to look for the shortest variability timescale. It should be closely related to the orbital period of the innermost stable circular orbit in the co-rotating case which is approximately 3.8 hours for the secondary, and 100 days for the primary. Due to Doppler boosting in the jet, variability time scales down to about 15 minutes may appear in the secondary jet and about 5 days in the primary jet. Therefore during secondary bursts the variability may be especially rapid. Possible evidence for this has may have been seen in the light curve of OJ 287 prior to the 1983 great outburst [43].

Further, considering that the semi-major axis of the system corresponds to an observed angular diameter of $\sim 0.01$ mas, it might be possible to follow the orbital motion of the binary in the sky, if the secondary is indeed bright enough. This requires observational capabilities with a spatial resolution at 10 microarcsecond level. This might be realized in the future e.g. with the GRAVITY instrument [44]. A direct detection would naturally confirm the binary nature of the OJ 287 system, but also allow correlating light curve events with the phase of the binary orbit.

A difficulty in investigating the secondary spin is the fact that the secondary is accreting material from a shear flow. Tentative arguments indicate that this would result in a torque working to align the secondary black hole spin in the opposite direction of the angular momentum of the accretion disk of the primary. The problem is additionally complicated by the manner of interaction with the matter to be accreted, i.e. the periodic impacts with an accretion disk. The impacts lead to a cloud of shocked gas, of which some part is accreted by the secondary, but in an amount and manner that is difficult to estimate analytically. In addition to secondary accretion events, both from the accretion disk and coronal cloud impacts, the magnetic field of the accretion disk of the primary likely affects the secondary brightness [45]. Thus as natural further work, ascertaining our estimates and the model for precursor bursts probably requires numerical simulations of the secondary accretion disk impacts, corona of the primary accretion disk and the effect of the magnetic field on the secondary brightness in different phases of the binary orbit.

\section{CONCLUSIONS}

We have discussed precursor bursts, a type of outburst of the supermassive binary black system OJ 287, and presented a model where the precursor bursts originate from the secondary component in the system. This requires the secondary black hole to have a high spin that is aligned with the spin of the primary black hole. We have argumented that the secondary is likely to have these properties due to the periodic interactions with the accretion disk of the primary, consistent with the binary model. We have further shown that the magnetic field in the system can be strong enough to have a significant effect on the secondary spin alignment. 
P. Pihajoki acknowledges the support of Turku University Foundation (grant no. 7642) and Magnus Ehrnrooth foundation (grant no. Ta2012n6).

\section{References}

[1] Sillanpää, A., Haarala, S., Valtonen, M. J., Sundelius, B. \& Byrd, G. G., ApJ 325, (1988) 628

[2] Sillanpää et al., A\&A 305, (1996) L17

[3] Sillanpää et al., A\&A 315, (1996) L13

[4] Lehto, H. J. \& Valtonen, M. J., ApJ 460, (1996) 207

[5] Sundelius, B., Wahde, M., Lehto, H. J. \& Valtonen, M. J., Blazar Continuum Variability, ASP Conf. Ser. 110, (1996) 99

[6] Sundelius, B., Wahde, M., Lehto, H. J. \& Valtonen, M. J., ApJ 484, (1997) 180

[7] Valtonen, M. J. et al., ApJ 729, (2011) 33

[8] Valtonen, M. J. et al., ApJ 709, (2010) 725

[9] Valtonen, M. J. \& Lehto, H. J., ApJ 481, (1997) L5

[10] Misner, C. W., Thorne, K. S. \& Wheeler, J. A., Gravitation (Freeman, New York 1973) 876

[11] Valtonen, M. J. et al., ApJ 742, (2011) 22

[12] Kidger, M. R. \& Takalo, L. O., IAU Circ. 5909, (1993) 1

[13] Kidger, M. R. et al., Intensive Monitoring of OJ287, eds. M. R. Kidger \& L. O. Takalo, Tuorla Observatory Rep. 174, (1994) 106

[14] Kidger, M. R. et al., Astron. Astrophys. Suppl. Ser. 113, (1995) 431

[15] Valtonen, M. J. et al., ApJ 646, (2006) 36

[16] Valtonen, M. J., ApJ 659, (2007) 1074

[17] Valtonen, M. J. et al., Cel. Mech. Dyn. Astr. 106, (2010) 235

[18] Mikkola, S. \& Aarseth, S., Cel. Mech. Dyn. Astr. 84, (2002) 343

[19] Pihajoki, P. et al., ApJ (2012) (in review)

[20] Bardeen, J. M., Nature 226, (1970) 64
[21] Thorne, K. S., ApJ 191, (1974) 507

[22] Press, W. H., ApJ 175, (1972) 243

[23] Lovelace, R. V. E., Rothstein, D. M. \& BisnovatyiKogan, G. S., ApJ 701, (2009) 885

[24] Beckwith, K., Hawley, J. F. \& Krolik, J. H., ApJ 707, (2009) 428

[25] Stella, L. \& Rosner, R., ApJ 277, (1984) 312

[26] Sakimoto, P. J. \& Coroniti, F. V., ApJ 247, (1981) 19

[27] King, A. R., Pringle, J. E. \& Livio, M. MNRAS 376, (2007) 1740

[28] Worrall, D. M. et al., ApJ 261, (1982) 403

[29] Valtonen, M. J. et al., ApJ 643, (2006) L9

[30] Bassani, L., Dean, A. J. \& Sembay, S., A\&A 125, (1983) 52

[31] Iwasawa, M. et al., ApJ 731, (2011) L9

[32] Bardeen, J. M. \& Petterson, J. A., ApJ 195, (1975) L65

[33] Rees, M. J., Nature 275, (1978) 516

[34] Natarajan, P. \& Pringle, J. E., ApJ 506, (1998) L97

[35] Scheuer, P. A. G. \& Feiler, R., MNRAS 282, (1996) 291

[36] Volonteri, M., Madau, P., Quataert, E., Rees, M. J., ApJ 620, (2005) 69

[37] Blandford, R.D. \& Znajek, R.L., MNRAS 179, (1977) 433

[38] Blandford, R. D. \& Payne, D. G., MNRAS 199, (1982) 883

[39] Meier, D. L., Koide, S. \& Uchida, Y., Science 291, (2001) 84

[40] King, A. R. \& Lasota, J. P., A\&A 58, (1977) 175

[41] Palenzuela, C., Garrett, T., Lehner, L. \& Liebling, S. L., Phys.Rev.D 82, (2010) 44045

[42] Palenzuela, C., Lehner, L. \& Liebling, S. L., Science 329, (2010) 927

[43] Valtaoja, E. et al., Nature 314, (1984) 148

[44] Eisenhauer, F. et al., The Messenger 143, (2011)16

[45] Neilsen, D. et al., Proc. Nat. Acad. Sci. 108, (2011) 12641 